\title{
Assessment of the Degree and Types of Dehydration in Libyan Children Suffering from Acute Diarrheal Diseases in Gharyan City
}

\author{
Aisha A Sehari* and Sana Taher Ashur \\ Faculty of Medicine, University of Tripoli, Tripoli, Libya \\ *Corresponding Author: Aisha A Sehari, Faculty of Medicine, University of Tripoli, \\ Tripoli, Libya.
}

Received: June 15, 2021

Published: June 30, 2021

(C) All rights are reserved by Aisha A Sehari and Sana Taher Ashur.

\begin{abstract}
Introduction: Across all ages, diarrheal disease causes more illnesses than any other ailment and is second only to pneumonia as the largest killer of children under five years. Children who survive an episode of diarrhea, but experience recurrent infections, are more likely to suffer from lifelong cognitive and physical impairments. Diarrheal disease is preventable and treatable. Deaths have declined in recent years-but children are still getting sick and surviving, facing the long-term consequences of repeated infections. New threats like COVID-19, antibiotic resistance, climate change, migration, and urbanization create new challenges and urgency. This work was planned to determine the prevalence of different type of dehydration clinically in acute diarrhea along with serum sodium level. Study of some co morbid factors were done.

Method: Retrospective review study of all patients aged 1 month to 60 months admitted to Gharrian Teaching hospital with acute diarrhea < 14 days duration from 2015 to 2018. Children with Dehydration were clinically classified as mild, moderate and severe according to WHO classification and depending on serum sodium level these studied children were also classified into isonatraemic--hyponatraemic----hypernatraemic dehydration. Complete blood picture (CBC), urea, blood sugar and arterial blood gases were done.

Results and Discussion: The total number of the children aged 1 month to 60 months admitted to Gharyan teaching Hospital complaining of acute diarrheal diseases during that period were 605 patients. The mean age of children was 11.7 (SD = 8.6) months, males represented $59.0 \%$ of the children patients. Of all acute diarrheal cases,157 (25.9\%) of children presented as severe degree, out of them 14 (8.9\%) were shocked), moderately dehydrated children were $439(72.6 \%)$ and $9(1.4 \%)$ were mildly dehydrated. Isotonic type was reported in 432 (71.4\%) of dehydrated children. hypertonic dehydration was reported in101 child (16.7\%) and hypotonic in 70 children patients (11.4\%).

Severe-shock presentation was more frequent among children who have hypertonic dehydration (60.4\%) than in those presented with hypotonic $(11.8 \%)$ or isotonic $(20.4 \%)$ dehydration and the type of dehydration showed a statistically significant association with the degree of the dehydration ( $\mathrm{p}<0.001)$. Interestingly, a statistically significant association between the degree of the dehydration and platelets count was reported. Shock presentation was more prevalent among children aged less than one year old (31.1\%) than in the older age groups, and age displayed a statistically significant association with the degree and severity of diarrhea ( $\mathrm{p}=$ 0.007). No statistically significant association was found between sex and the severity of diarrhea. The median urea difference was statistically significant $(\mathrm{p}<0.001)$.

Conclusion: So, we conclude that acute diarrhea is still a major problem and severe hypertonic dehydration is still high in our children, so we recommend early diagnosis and uses of ORS, fluid and education of society about it, to avoid complications. Support for research and development of new drugs and vaccines can accelerate an end to the crisis.
\end{abstract}

Keywords: Acute Diarrhea; Type of Dehydration; Degree of Dehydration; Thrombocytopenia 


\section{Introduction}

Acute diarrhea is the most common gastrointestinal disorder in children, and causes many complications [1-4]. It is manifested as sudden episode of loose watery stool which is generally 3 or more episodes per day and last for seven to less than 14 days. most episodes of acute diarrhea are infectious and caused by a variety of viruses and bacteria [5,6]. Despite substantial reductions in deaths among children under five years-from more than 1 million to roughly 500,000 each year in the last 15 years-diarrhea remains one of the deadliest threats young children face. It is responsible for about nine percent of all worldwide deaths in young children $[7,8]$. More than half of these cases are in Africa and South Asia, where bouts of diarrhea are more likely to result in death or other severe outcomes. The incidence of diarrheal diseases varies greatly with the seasons and a child's age. The youngest children are most vulnerable: Incidence is highest in the first two years of life and declines as a child grows older $[9,10]$.

Diarrheal disease is caused by germs (both viral and bacterial) and parasites that are spread from the waste of an infected person to the mouth of another person through contaminated water, food, objects, or hands. During a diarrhea episode, fluids are depleted. Diarrhea becomes deadly when it results in dehydration that goes untreated [11-14].

The top six pathogens responsible for acute diarrhea are Shigella, Enterotoxigenic E. coli, Campylobacter, Rotavirus, Adenovirus and Cryptosporidium [15].

The main aim of treatment is to correct the fluid and metabolic disturbance urgently to avoid more complications and this is usually done by oral rehydration salts and intravenous fluid therapy. Worldwide only $20 \%$ of children with acute diarrhea receive appropriate Oral rehydration therapy $[17,18]$.

Good nutrition and continuing of breast feeding during the diarrheal episodes are very important measures to improve the immunity and enhance the recovery of the affected children $[19,20]$.

\section{Aims and Objectives:}

1. To find the prevalence of different degrees of dehydration (mild, moderate and severe) clinically in acute watery diarrhea in children admitted to Gharryan Teaching Hospital up to 5 years of age.

2. To determine the prevalence of different types of dehydration (isonatraemic, hyponatraemic, hypernatraemic) depending serum sodium level.
3. To study the change in serum sodium level according to severity of dehydration.

4. To find the relation between degree of dehydration and platelets count as a first sign of disseminated intravascular coagulation (DIC).

\section{Materials and Methods}

Retrospective study was conducted on 605 children complaining of acute diarrhea admitted to Gharryan Teaching hospital from January 2015 to December 2018.

Sample collection: $5 \mathrm{ml}$ of venous blood was collected at admission and before treatment.

\section{Inclusion criterion:}

a. All children aged 1 month to 60 months of age, of either sex having history of acute onset of watery diarrhea not more than 14 days of duration.

b. All cases must have dehydration.

\section{Exclusion criterion:}

a. Patients who had bleeding disorder.

b. Patients with metabolic disease/chronic medical condition which can alter serum electrolytes like nephrotic syndrome, renal failure.

Detailed history and thorough clinical examination of cases included in the study was done. Along with general information and presenting illness special emphasis was on any given treatment or fluid, diet taken prior to admission. General physical examination done and type of dehydration assessed clinically according to WHO chart.

\section{Data analysis subsection}

Data analysis was done using the statistical package for social sciences (SPSS) version 26. Frequency and percentage were used to summarize the categorical variables. Mean and standard deviation and median and interquartile range were used to describe the numerical variables as appropriate to their distribution. The bivariate associations between the outcome variable; severity of diarrhea (mild-moderate versus severe-shock), and the study variables were tested using Chi square test, independent $t$ test and Mann Whitney test as appropriate to the type and distribution of data. A significance level of $p$ less than $0.5 t$ is like if we study the association between a part to all sort of compound variable. 


\section{Results}

After preliminary data exploration, 605 cases where available for the final analysis, with missing data on any variable was below $0.8 \%$.

Table 1 shows the socio-demographic, clinical and laboratory characteristics of the children in this study sample.

\begin{tabular}{|l|c|c|c|}
\hline \multicolumn{1}{|c|}{ Variable } & f & (\%) & Range \\
\hline Age & & & \\
\hline$<1$ years & 384 & $(63.5)$ & \\
\hline 1 - 2 years & 171 & $(28.3)$ & \\
\hline$>2$ - 5 years & 50 & $(8.3)$ & \\
\hline Age (months) (mean \pm SD) & 11.7 & \pm 8.6 & $1-60$ \\
\hline Sex & & & \\
\hline Male & 357 & $(59.0)$ & \\
\hline Female & 243 & $(40.2)$ & \\
\hline Degree of dehydration & & & \\
\hline Mild & 7 & $(1.2)$ & \\
\hline Moderate & 439 & $(72.6)$ & \\
\hline Severe & 143 & $(23.6)$ & \\
\hline Shocked & 14 & $(2.3)$ & \\
\hline Type of dehydration & & & \\
\hline Hypotonic & 68 & $(11.2)$ & \\
\hline Isotonic & 432 & $(71.4)$ & \\
\hline Hypertonic & 101 & $(16.7)$ & \\
\hline Platelets Count & & & \\
\hline Normal & 1368 & $(77.4)$ & \\
\hline Thrombocytopenia & 4.3 & \pm 0.7 & $1.7-6.8$ \\
\hline Urea (median, IQR*) & & & \\
\hline Sodium (mean \pm SD) & $(92.6)$ & \\
\hline Potassium (mean \pm SD) & & \\
\hline
\end{tabular}

Table 1: Socio-demographic, clinical and biomedical pattern of acute diarrhea.

*: IQR= Interquartile Range.

The mean age of the children was 11.7 ( $\mathrm{SD}=8.6$ ) months. Age ranged between 1 to 60 months, with more than half of the children aged below one-year old (63.5\%).

Both sexes were represented in the sample, with the predominance of boys (59.0\%).

With regard to the degree of dehydration, $23.6 \%$ of children presented as severe degree, and out of them $2.3 \%$ were shocked.
Interestingly, $26.0 \%$ of all diarrhea cases had thrombocytopenia.

Blood urea ranged between 2 to $231 \mathrm{mg} / \mathrm{dL}$, with a median urea level of $19(\mathrm{IQR}=9-34) \mathrm{mg} / \mathrm{dL}$.

Table 2 shows the distribution of the degree of dehydration, (mild-moderate versus severe-shocked) by the socio-demographic characteristics and clinical and laboratory profiles as well as their bivariate associations. Concerning the socio-demographic characteristics, severe-shock presentation was more prevalent among children aged less than one year old (31.1\%) than in the older age groups, and age displayed a statistically significant association with the degree of dehydration ( $p=0.007)$. The percentage of children presented with severe diarrhea- shock among males $(26.6 \%)$ was comparable to that among females (25.5\%) and no statistically significant association was found between sex and the severity of diarrhea.

Severe-shock presentation was more frequent among children who have hypertonic dehydration (60.4\%) than in those presented with hypotonic $(11.8 \%)$ or isotonic $(20.4 \%)$ dehydration. The type of dehydration showed a statistically significant association with the degree of degree of dehydration $(\mathrm{p}<0.001)$. Interestingly, a statistically significant association between the degree of dehydration and platelets count was reported $(\mathrm{P}=0.008)$, severely dehydrated (shocked), presentation was more in children who have thrombocytopenia (35.0\%) than in those with had normal platelets count $(23.4 \%)$.

The median blood urea level was higher among children presented with severe degree of dehydration (shocked 41, IQR = 61.527) compared to that among children with mild-moderate diarrhea $(15, I Q R=26-9)$, and the median urea difference was statistically significant $(\mathrm{Z}=11.474, \mathrm{p}<0.001)$. Likewise, the mean blood sodium level was higher in the severe-shock group (Mean $=148.2, \mathrm{SD}$ $=12.6)$ than in the mild to moderate diarrhea group $(140.3, \mathrm{SD}=$ 7.2), and the mean difference was statistically significant $(t=7.429$, $\mathrm{p}<0.001$ ). However, although there was a difference in the mean blood potassium level across the two groups of diarrhea severity, the mean difference was not significant ( $p>0.05$ ).

\section{Discussion}

Globally, diarrheal diseases account for almost a fifth of all deaths in children aged under 5 years which is equal to 2.2 million deaths annually [1-9].

Pediatric diarrhea is a costly disease in terms of direct (and indirect) monetary costs to each community, and it is a cause of emotional trauma for the child and the parents $[10,11]$. 


\begin{tabular}{|c|c|c|c|c|c|c|}
\hline \multirow[b]{2}{*}{ Variables } & \multicolumn{4}{|c|}{ Severity of diarrhea } & \multirow[t]{2}{*}{$\chi^{2 \mathrm{a}}$} & \multirow[t]{2}{*}{$\mathbf{P}$} \\
\hline & \multicolumn{2}{|c|}{ Mild to moderate } & \multicolumn{2}{|c|}{ Severe-Shocked } & & \\
\hline & \multicolumn{2}{|c|}{ f (\%) } & \multicolumn{2}{|c|}{ f (\%) } & & \\
\hline${ }^{\dagger}$ All $(n=603)$ & 446 & $(73.7)$ & 157 & $(26.0)$ & & - \\
\hline \multicolumn{7}{|l|}{ Age } \\
\hline$<1$ years & 267 & $(69.9)$ & 115 & $(30.1)$ & & \\
\hline 1 - 2 years & 141 & $(82.5)$ & 30 & $(17.5)$ & & \\
\hline$>2-5$ years & 38 & $(76.0)$ & 12 & $(24.0)$ & 9.795 & $0.007^{*}$ \\
\hline \multicolumn{7}{|l|}{ Sex ${ }^{a}$} \\
\hline Male & 262 & 73.4 & 95 & 26.6 & & \\
\hline Female & 181 & 74.5 & 62 & 25.5 & 0.090 & 0.764 \\
\hline \multicolumn{7}{|l|}{ Type of dehydration } \\
\hline Hypotonic & 60 & 88.2 & 8 & 11.8 & & \\
\hline Isotonic & 344 & 79.6 & 88 & 20.4 & & \\
\hline Hypertonic & 40 & 39.6 & 61 & 60.4 & 76.146 & $0.000^{*}$ \\
\hline \multicolumn{7}{|l|}{ Platelets Count } \\
\hline Normal & 357 & 76.6 & 109 & 23.4 & & \\
\hline Thrombocytopenia & 89 & 65.0 & 48 & 35.0 & 7.457 & $0.008^{*}$ \\
\hline Urea & 15 & $17(26-9)$ & 41 & $(61.5-27)$ & $11.474^{\mathrm{b}}$ & $0.000^{*}$ \\
\hline Sodium & 140.3 & \pm 7.2 & 148.2 & \pm 12.6 & $7.429^{c}$ & $0.000^{*}$ \\
\hline Potassium & 4.2 & \pm 0.6 & 4.3 & \pm 0.7 & $0.975^{c}$ & 0.330 \\
\hline
\end{tabular}

Table 2: Comparison of socio-demographic characteristics, type and biomedical parameters by the severity of diarrhea.

${ }^{\dagger}$ Missing data on degree of diarrhea on 2 cases, ${ }^{*} \mathrm{p}<0.05$, ${ }^{\mathrm{a}} \mathrm{Chi}$ square test statistic, ${ }^{\mathrm{b}} \mathrm{Z}$ statistic (Mann-Whitney -U test). ${ }^{\mathrm{c} t}$ statistic (independent).

This retrospective study was conducted on 605 Libyan children suffering of acute diarrhea with mild (7), moderate (439) and severe (157) dehydration missing data about the degree of dehydration were reported on 2 children which was statistically insignificant. All admitted children were investigated for electrolytes profile, blood urea, sugar and hematological changes before the start of either oral or infusion therapy.

The mean age of children was 11.7 (SD = 8.6) months, males represented $59.0 \%$ of the children patients.

Of all acute diarrheal cases, 157 (25.9\%) of children presented as severe degree, out of them 14 (8.9\%) were shocked), moderately dehydrated children were 439 (72.6\%) and 7 (1.2\%) were mildly dehydrated. No statistically significant association was found between sex and the degree of dehydration.

Isotonic type was reported in 432 (71.4\%) of dehydrated children. hypertonic dehydration was reported in 101 (16.7\%) and hypotonic in 68 (11.2\%).
Mean serum sodium was significantly higher in Severe-shock presentation, $(60.4 \%)$ than in those presented with hypotonic (11.8\%) or isotonic $(20.4 \%)$ dehydration, and the type of dehydration showed a statistically significant association with the degree of the dehydration, ( $p<0.001)$. Hypernatremia was reported in Libyan infant and children ( $<5$ years) to be $10 \%$ in Ben ghazi and 2\% in Algalaa Hospital.

In a recent change, WHO now recommend reduced osmolarity ORS, with, $75 \mathrm{mEq}$ per L of sodium and $75 \mathrm{mmol}$ per L of glucose.

Caregivers should be taught how to prepare and how to be given either by a syringe or a spoon depending on the age of the child. as these points misuse were one of expected causes of high prevalence of hypernatremia in Libyan infants in a previous study plus high solute load of water in some area of my country [21,22].

Regarding serum potassium there was a difference in the mean blood potassium level across the two groups of diarrhea (mild to 
moderate and severe), the mean difference was not significant ( $\mathrm{p}$ $>0.05)$. However, high potassium (> $5.5 \mathrm{mEq} / \mathrm{L})$ was obtained in seventeen children patient $(2.8 \%)$ in the present work. This finding could be attributed to hemoconcentration, intracellular shift of excess hydrogen ions replacing the intracellular potassium and lastly due to prerenal failure (hypoperfused kidneys in dehydration). Acidosis $(\mathrm{pH}<7.33$ ) was detected in all severely dehydrated children (157) patient out of 605 children (25.9\%) and most of moderately dehydrated children. Usually, every dehydrated case is considered potentially acidotic by different investigations [8].

The median urea difference was statistically significant $(\mathrm{p}<$ $0.001)$.

Interestingly, a statistically significant association between the degree of the dehydration and platelets count was reported. This was approved in a previous study [23]. Shock presentation was more prevalent among children aged less than one year old $(31.1 \%)$ than in the older age groups, and age displayed a statistically significant association with the degree and severity of diarrhea $(\mathrm{p}=0.007)$.

Respiratory tract infection was reported in 38 patients as a co morbid factor in these studied children.

One female child died aged 5 months, with severe degree, hypertonic, dehydration, her serum sodium was $178 \mathrm{mEq} / \mathrm{L}, \mathrm{K} 2.6$ $\mathrm{mEq} / \mathrm{L}$, Blood urea was $147 \mathrm{mg} / 100 \mathrm{ml}$.

\section{Conclusion}

So, we can conclude:

- That acute diarrheal diseases still a serious disease to children especially in young age group less than two years of age although it partially a preventable disaster.

- Hypertonic dehydration is still a serious complication of acute diarrhea but its prevalence is less than before compared to our previous studies.

- No broad knowledge about the causes of diarrhea in our population which needs a further studies and hard research work.

\section{Recommendations [24-33]:}

- We recommend early diagnosis and uses of reduced osmolarity oral rehydration therapy (ORS), for children with mild to moderate dehydration due to acute gastroenteritis, and education of society about it for its use, benefits and preparation, to avoid complications.
- Hand washing with soap is an effective method for preventing episodes of gastroenteritis.

- All children should receive an oral live, attenuated rotavirus vaccine to reduce the incidence of hospitalization, severe gastroenteritis, and death from rotavirus infection.

- Breastfeeding reduces the incidence of acute gastroenteritis and hospitalization from diarrheal disease in young children.

- Support for research and development of new drugs, zinc supplement, using probiotics, proper sanitation and hand washing can prevent, cure, and accelerate an end to the crisis.

\section{Bibliography}

1. UNICEF/WHO. Diarrhea: Why children are still dying and what can be done. Geneva/New York: UNICEF, WHO (2009).

2. World Health Organization/The United Nations Children's Fund (UNICEF) (2013).

3. Elliott EJ. "Acute gastroenteritis in children". British Medical Journal 334.758 .3 (2007): 35-40.

4. Van den Bruel A., et al. "European Research Network on Recognising Serious Infection investigators. Diagnostic value of clinical features at presentation to identify serious infection in children in developed countries: a systematic review". Lancet 375.9717 (2010): 834-845.

5. Bhutta ZA., et al. "Interventions to address diarrhea and pneumonia deaths equitably: what works and at what costs?" Lancet (2013).

6. Graeme L Barnes., et al. "Etiology of Acute Gastroenteritis in Hospitalized Children in Melbourne, Australia, from April 1980 to March 1993". Journal of Clinical Microbiology 1 (1998): 133-138.

7. World Health Organization. The treatment of diarrhea: a manual for physicians and other senior health workers (2005).

8. Sperling MA. "Diarrhea and diarrhea in developing countries". Pehramn R, Kliegman R, Nelson W, Vaughan V, editions. Nelson Text book of paediatric $20^{\text {th }}$ edition Philadelphia, WB Saunders Company 2 (2016): 1854-1874.

9. Fischer Walker CL and Black RE. "Zinc for the treatment of diarrhoea: effect on diarrhoea morbidity, mortality and incidence of future episodes". International Journal of Epidemiology 39.1 (2010): i63-i69. 
10. Parashar UD., et al. "Diagnosis, management, and prevention of rotavirus gastroenteritis in children". British Medical Journal 347 (2013): f7204.

11. UNICEF/WHO. Diarrhoea: Why children are still dying and what can be done". New York, UNICEF (2009).

12. UNICEF. Pneumonia and diarrhoea: Tackling the deadliest diseases for the world's poorest children". New York, UNICEF (2012).

13. Lamberti LM., et al. "Breastfeeding and the risk for diarrhea morbidity and mortality". BioMed Central Public Health 11.3 (2011): S15.

14. UNICEF/WHO. Progress on drinking water and sanitation: 2012 update". New York, UNICEF (2012).

15. United Nations Resolution A/RES/58/217. International decade for action 'Water for life',2005-2015 (2013).

16. Aisha A Sehari., et al. "Acute Diarrhea and Types of Dehydration in Libyan Children". Acta Scientific Paediatrics 4.4 (2021): 2581-883.

17. King CK., et al. "Centers for Disease Control and Prevention. Managing acute gastroenteritis among children: oral rehydration, maintenance, and nutritional therapy". MMWR Recommendations and Reports 52.16 (2003): 1-16.

18. Duggan C., et al. "Centers for Disease Control and Prevention. The management of acute diarrhea in children: oral rehydration, maintenance, and nutritional therapy". MMWR Recommendations and Reports 41.16 (1992): 1-20.

19. Duijts L., et al. "Prolonged and exclusive breastfeeding reduces the risk of infectious diseases in infancy". Pediatrics 126.1 (2010): e18-e25.

20. Quigley MA., et al. "Breastfeeding and hospitalization for diarrheal and respiratory infection in the United Kingdom Millennium Cohort Study". Pediatrics 119.4 (2007): e837-e842.

21. BEN Halim MM. "Gastrointestinal in Tripoli children Hospital. Analysis of 1249 Hospital Admissions, Alfateh University, Libya (1991).

22. ARAB Libyan Maternal and Child Health Survey, League of Arabs States 1996 (1997).

23. Aisha A Sehari., et al. "Madamba Associating Disseminated Intravascular Coagulation with Clinical and Laboratory Evaluations of Pediatric Patients with Acute Gastroenteritis admit- ted at Gharyan Central and Teaching Hospital, Libya". Clinical Research and Clinical Case Reports 1.1 (2016).

24. Lazzerini M and Wanzira H. "Oral zinc for treating diarrhoea in children". Cochrane Database of Systematic Reviews 12 (2016): CD005436.

25. Clasen TF., et al. "Interventions to improve water quality for preventing diarrhoea”. Cochrane Database of Systematic Reviews 10 (2015): CD004794.

26. Clasen TF., et al. "Interventions to improve disposal of human excreta for preventing diarrhoea". Cochrane Database of Systematic Reviews 6 (2010): CD007180.

27. Aiello AE., et al. "Effect of hand hygiene on infectious disease risk in the community setting: a meta-analysis". American Journal of Public Health 98.8 (2008): 1372-1381.

28. Ejemot-Nwadiaro RI., et al. "Hand washing promotion for preventing diarrhoea”. Cochrane Database of Systematic Reviews 9 (2015): CD004265.

29. Parashar UD., et al. "Prevention of rotavirus gastroenteritis among infants and children. Recommendations of the Advisory Committee on Immunization Practices (ACIP)". MMWR Recommendations and Reports 55.12 (2006): 1-13.

30. Committee on Infectious Diseases; American Academy of Pediatrics. Prevention of rotavirus disease: updated guidelines for use of rotavirus vaccine". Pediatrics 123.5 (2009): 14121420 .

31. Lawrence RA and Lawrence RM. "Breastfeeding: A Guide for the Medical Profession". 8th edition. Philadelphia, Pa.: Elsevier (2016): 146-193.

32. Del Piano M., et al. "Is microencapsulation the future of probiotic preparations? The increased efficacy of gastro-protected probiotics". Gut Microbes 2.2 (2011): 120-123.

33. Riaz QU and Masud T. "Recent trends and applications of encapsulating materials for probiotic stability". Critical Reviews in Food Science and Nutrition 53.3 (2013): 231-244.

\section{Volume 4 Issue 7 July 2021}

(C) All rights are reserved by Aisha A Sehari and Sana Taher Ashur. 